\title{
11 Introduction to Part III
}

\author{
Jorgen Bruhn and Beate Schirrmacher
}

What is now known as intermedial studies developed from interart studies, which explored the relation between literature, art, music and film. Due to this historical development, intermedial research has been mostly interested in analysing artistic media products, and the analytical methods that we presented in Part II were primarily exploring artistic communication. This third part intends to widen the scope of intermedial studies in several ways. First, we want to stress how intermedial theory, although developed in the context of artistic communication, is not restricted to analysing works of art. Instead, we demonstrate in different ways how intermedial analysis can be used to understand the combinations and transformations of all kinds of media types. In different ways, this third part means to highlight the importance of interaction across theoretical fields, across media borders and in the digital sphere in particular. We therefore take a closer intermedial look at the workings of social media and of computer games. We also present an intermedial approach to performance that can combine the focus on the body from performance studies with an intermedial analysis of media types. We use the spatiotemporal modality as a lens to describe the interaction of different kinds of spaces and bodies in theatre and performance. Furthermore, we focus on transmedial phenomena that are not restricted to only one qualified media type but which cross conventional media borders and may need new productive methods to analyse the way communication is transformed in these processes. Therefore, we explore several transmedial storyworlds in popular culture. We also claim that understanding the social challenges of communication needs an intermedial approach and a transmedial perspective. This is the reason why we explore how intermedial analysis contributes to understanding societal challenges, here exemplified by the communication of climate change, on the one hand, and the destructive spreading of disinformation, on the other.

DOI: $10.4324 / 9781003174288-14$ 